\title{
Issues in Techno-Organisational Development Methods for Complex Manufacturing Environments
}

\author{
António Brandão Moniz \\ UNINOVA - Quinta da Torre, 2285 Monte de Caparica, \\ Portugal \\ António Lucas Soares \\ INESC - Rua José Falcão 110, 4300 Porto, Portugal and \\ FEUP/DEEC, Univ. of Porto, $R$ dos Bragas 4099, Porto Codex, \\ Portugal. \\ email: abm@uninova.pt,als@fe.up.pt
}

\begin{abstract}
This paper presents a reference position on the relation between manufacturing systems and work organisation strategies. It raises some issues and advances a methodological framework in the development of information/manufacturing systems and work organisation design methods, as to reach a balanced influence of the technology, organisation and people aspects in Complex Manufacturing Environments (CME). In this sense it is not presenting research results but, with the underlying goal of implementing a laboratory environment for the study of technical and organisational developmental aspects of CMEs, there are overviewed the R\&D requirements for an interdisciplinary approach involving Computer and Social Sciences. The main scientific areas analysed are Enterprise Integration and Work Organisation, focusing on the methodological aspects of each one.
\end{abstract}

\section{Keywords}

Techno-organisational development, socio-technical systems, enterprise integration, work organisation.

\section{INTRODUCTION}

The success of the information technology (IT) appliance as support to the activities undertaken in manufacturing systems is closely linked to the analysis of human factors, being reflected in the strategies and methods of work organisation, ergonomic aspects of workplace design and in an organisational analysis that includes also social relations and enterprise culture. The interaction between the technological and organisational development assumes an 
increasing importance if we consider that it is now irreversible the way towards complex manufacturing environments (CME). With CME we mean work environments that demand high levels of abstract reasoning for the performance of the organisational tasks, resulting from new organisational forms, leading to work enrichment and the use of advanced IT tools. These "environments" are in a way the materialisation of the concept "simple organisations and complex jobs" (De Sitter and Den Hertog, 1990). It is well known that the technological and organisational innovation are crucial processes that together promote higher competitiveness and better work conditions. These processes can not be decoupled when we foresee a sustained development perspective and, therefore, the corresponding processes of design and implementation are extremely interdependent. In this paper we will raise some issues and outline a methodological framework aiming at contribute to the interdisciplinary development of CMEs through a synthesis of methodologies coming from the areas of Engineering (Computing, Production) and Social Sciences (Sociology, Psychology, Management). The ideas presented here are also intended to launch some discussion on the materialisation of such interdisciplinary approaches (Latniak, 1993), being in the form of national or trans-national R\&D projects or in the form of academic research lines. The proposed approach combines a macro-analysis, where the interrelations between enterprise integration and the organisation and work structures are investigated and synthesised, with a micro-analysis developing new concepts of techno-organisational design based on a laboratory environment. The objectives of this approach can be framed in two streamlines:

- the characterisation of the organisational, social and individual influence of state-of-the-art development methodologies for information systems and enterprise integration, including also IT platforms, architectures and applications in the area of advanced manufacturing management systems and,

- the synthesis of criteria and conceptual methods of techno-organisational design, having a prototype (laboratorial setting) as an experimentation and development platform, aiming at select the "best methods" and verify its applicability in a sectorial or national context.

\section{A METHODOLOGICAL FRAMEWORK}

The aim of this approach is the socio-technical evaluation of the appliance of human-centred systems to manufacturing industries with the objective of demonstrating and assessing the superiority of these methods over the conventional approaches to automation. This evaluation must not be merely an economic one (cost analysis, accounting), but will include mostly social variables, such as: working conditions, development of indirect operations and its effect on the tacit knowledge, skilled work in automated production systems, autonomous working groups, de-centralisation of decision making. The Engineering and Social Sciences literature is not yet developed in this field, and there are some experiments in this scientific areas that use an intensifying collaboration with the Computer Sciences, Quality and Production Engineering, and Social Sciences approaches. Some ESPRIT and BRITE projects can be used as reference frameworks to this collaboration, in particular those with special references to Social Sciences i.e., the ESPRIT 1199/1217 "Human-Centred CIM Systems", ESPRIT 5564 


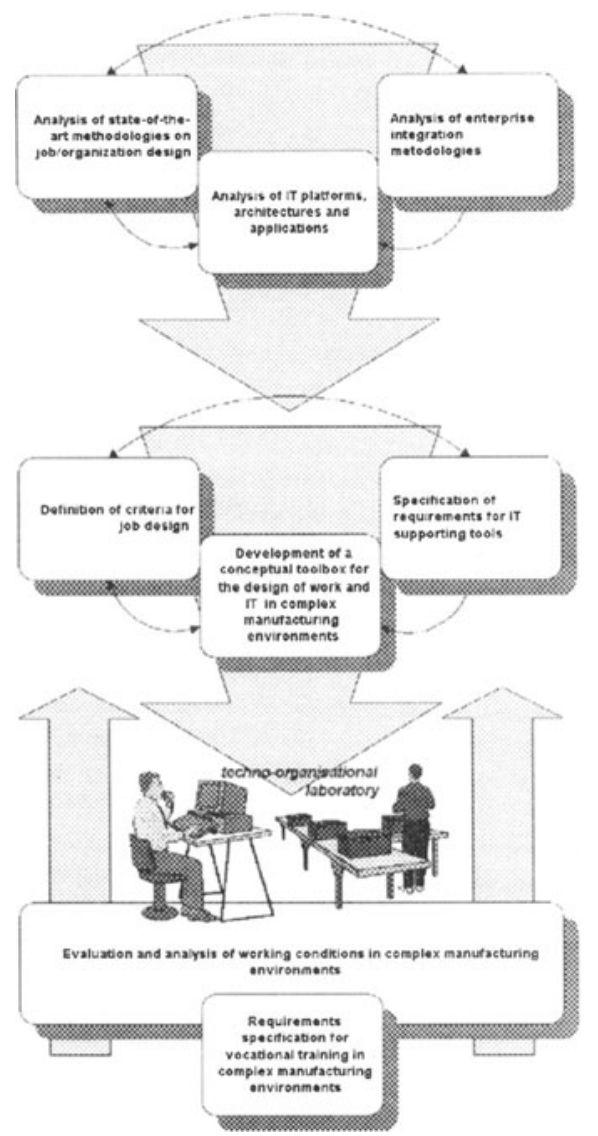

Figure 1: A framework for techno-organisational development in a laboratory environment.

"Integrated Design and Evaluation of Assembly Lines within CIM", or the BRITE projects 1381 (on interactive knowledge based shopfloor control systems), 3302 (on Decision Support Systems) or 3345 (on Flexible Production Groups), or even the ESPRIT exploratory action 5603 on "Joint Technical and Organisational Design of CIM systems for SMEs". At the same time, it has been recently recognised that the human-centred systems concepts, and the Human and Computer Integrated Manufacturing concepts, are more and more determinants for the correct efficiency and performance of flexible and automated production systems. It is necessary then to analyse, design and evaluate integrated socio-technical systems, specially the new systemic relation between the organisation, the technical system and the social and economical environment. Some of those issues have been analysed, and there is a trend to approach this new problems through some FAST projects, namely the ones related to APS-Anthropocentric Production Systems, and the FINE-The Future of Industry in Europe, that involved even colleagues from the United States and Japan. Thus, the general idea 
under this approach is the development of a techno-organisational test bed, using a real FMS as a demonstrator and producing several interfaces with IT criteria and architectures (see Figure1). The goal is the development of methodologies for job design in complex environments/organisations.

This project aims the analysis of social implications of use of IT platforms in advanced manufacturing systems, using a simulated situation that will be assessed. In this sense this approach develop some new job design methodologies in reference to organisational networks (working groups, production cells) and with IT support tools. These methodologies are to be implemented in a technical environment where alterations to the lay-out or other technical re-configurations can be done, ensuring a global prototyping activity in a socio-technical framework of scientific and technological development.

\section{ENTERPRISE INTEGRATION: A SOCIO-ORGANISATIONAL VIEW}

There is a growing diffusion in the scientific technical community linked to industry and in some cases in the industry itself of the concepts of Enterprise Integration (EI), consolidated by the so called Enterprise Reference Architectures and Methodologies (ERA\&M). These concepts are intended to cope with the extreme complexity of a full enterprise integration, and claim to provide multidisciplinary frameworks to take into account the economic, social and human points of view besides the technical one. What actually comes up from the academic discussion is that, safeguarding some exceptions, things run in an exclusively technical dimension sometimes punctuated by technical-organisational insights or rather superficial social and individual considerations. It is likely that these concepts became paradigmatic (a little like the CIM concepts in the early 80 's) so it is necessary to evaluate them in terms of hindrances and opportunities in the promotion of a better match between organisations, people and technology. A short review of the state-of-the-art in ERA\&M is given below. Enterprise Integration is referred in the literature as a global approach to co-ordinate in an integrated way all the components of an enterprise in order to improve its overall performance. To undertake an EI project, Enterprise Reference Architectures and Enterprise Integration Methodologies are necessary to make an effective use of methods, models and tools, and to cope with the underlying complexity of the integration process. There are today three ERA\&M that claim to deal with the "structural arrangement or organisation of the development and implementation of a project or a program such as manufacturing or enterprise integration or other enterprise development program" (Williams, 1993):

- Open Systems Architecture for Computer Integrated Manufacturing - CIMOSA - as developed by the European CIM Architecture Consortium (AMICE) since 1984 under ESPRIT projects $668,2422,5288$ of the EU and being now an European prenorm known as CEN ENV 40003.

- GRAI - GIM - the GRAI Integrated Methodology as developed by the GRAI Laboratory of the University of Bordeaux in France. This work resulted from production management studies initiated in the GRAI laboratory and has taken its current form since about 1984.

- Purdue Enterprise Reference Architecture (PERA) and the related Purdue Methodology as 
developed at Purdue University and was part of the work of the Industry-Purdue University Consortium for CIM setup since 1989.

These ERA\&M are analysed in a socio-organisational light, and some conclusions taken into account in the development and running of the techno-organisational prototype. The intention of this review of the enterprise development methodologies state-of-the art is to draw some conclusions about their capability to deal with socio-organisational variables in the techno-organisational development process. The main sources of these variables are the SocioTechnical Systems Theory (STST), the sociological Strategic Analysis Theory (SAT) and the Work Organisation Concepts (WOC). The objective of dealing with socio-organisational variables is to make possible the reasoning about the technical, organisational and people aspects in complex manufacturing environments development. These aspects include: structural and functional interdependence, organisational units qualification and regulation, action systems, uncertainty zones, skills variety, task identity and autonomy. The ERA\&M will be looked according the above view, trying to identify the enabling features as well as the obstacles in:

1. representing the techno-organisational reality - inclusion, absence or possible inference of the following socio-organisational variables: structure (functions, tasks, resources), information network, decision process, actor, authority, responsibility, power relation, skills;

2. facilitating the development actors participation - inclusion or absence of procedures, guidelines or measures to promote users peer participation in the development process.

The analysis framework is based on the two above referred capability classes. Additionally, it will be identified if those features are intrinsic or extrinsic, i.e., if the capabilities exist, whether they are inherent to the methodology or there is a need of plugging-in new models and tools to make use of them.

\subsection{Integration Architectures, Platforms and Applications}

The definition of an Integration Architectures to model the different activities of an enterprise usually is not done considering the (existing) humans activities, behaviours and expertise. In fact, that definition is oriented according to the computational and production resources requirements of the manufacturing system, and the humans assigned to "roles" not covered by them. In other words, the humans should adapt themselves to the "imposed" interfaces and planned processes/activities, regardless how this interfere in their motivation and work quality. The evaluation of IT platforms, architectures and applications to connect organisational networks in complex manufacturing environments aims at making an analysis of the integration and enterprise execution architectures development, focusing on how that desired human oriented approach can be incorporated into such developments.

\section{INTEGRATION STRATEGIES}

Integration strategies is a concept being used mainly in Computer Sciences and Production Engineering, and it is not been sufficiently assessed by the Social Sciences (i.e., Sociology, 
Psychology, Management). Manufacturing enterprises are willing to articulate their internal activities in order to rationalise the production systems using the general concept of "lean production" or "concurrent engineering". Within this strategy, the use of CIM systems is one of most tried solutions for those firms that are inserted in the globalisation processes with flexibilisation of manufacturing needs. When trying to optimise the process flow which compose a production system there are tools that facilitate it. When job designers want to rationalise the equipment, layout and task structure, they need to apply any method or methodology. The transformation flow chart is used for job designers when they attempt to conceptualise a production system. This tool shows the different operations which the product or service is transformed from one stage into another. Each operation includes decisionmaking, calculation, setup, positioning and so on. So it can be used to integrate different activities, units and work teams. Thus, the use of integration procedures is a production need, but normally is not accompanied by organisational changes. The social sciences literature demonstrates the need to stress in first place these change procedures in order to make possible the other integration strategies. It is not envisaged only the integration strategy at single work places or work groups levels. This task was developed at previous levels, where some conclusion should be made, about for example the so-called "object oriented" production design i.e., a group of products is to be produced in full responsibility by one work group.

\section{WORK ORGANISATION CONCEPTS}

Job design involves, for most researchers, some specific sets of decisions, such as:

- decisions on what tasks will be performed by operators;

- decisions on how these tasks can be grouped together and assigned to individuals;

- decisions on how those individuals can relate each other for co-ordination purposes;

- decisions on rewards and performance evaluation.

One of the goals in this methodological area is to bring together the ideas and criteria of the different actors involved. It is assumed that the criteria for job design in complex manufacturing systems are not restricted to the individual level, but are also related to the organisational and societal levels, taking into account the socio-technical and socio-economic performance of the system. It is necessary not only to gain criteria, but the intention would be to provide:

- further information on the effects of the principles on job design in complex manufacturing systems,

- a detailed reasoning why these principles are useful in developing efficient and effective organisation on the one hand and "good jobs" on the other,

- information about the developments of human capacities and skills in terms of human capital perspective.

The existing conceptual methods for the design of work in complex manufacturing envi- 
ronments have to be evaluated from the viewpoint of Social Sciences. As a result, selected methods (integrating structurally-oriented and process-oriented elements) have to be discussed and synthesised to provide a kind of "contemporary" or "state-of-the-art" method which can be applied in practice. Another important aspect is the establishment of influence lines between these methods and the enterprise integration methodologies. There is a need to devise combined approaches where work design outcomes directly induce the software applications development.

\section{THE TECHNO-ORGANISATIONAL SIMULATION LABORATORY}

The evaluation of the appliance of human-centred systems to manufacturing industries, using the socio-technical methodologies is one of the central tasks of this approach. The objective of demonstrating and assessing this task is to prove and underline the superiority of this method over the conventional approaches to automation. Thus, the analysis of the limits and obstacles of such conventional approaches as well as the testing of new tools and methods implies the development and running of a techno-organisational prototype. A FMS laboratory as the technical environment, together with people (professionals or students) as the organisational actors can provide a live laboratory for the assessment of new concepts. These ideas are in line with the subject of games in production management (Riis et al., 1993). A real test of the tools and ideas generated in initial tasks in a practice environment can be presented as a "simulated methodology". In this approach the simulation process uses a real infrastructure described in the next sub-task, but the usual criteria for simulation process can be used. Simulation-based approaches, although important as a design step, are not sufficient to understand and evaluate all behavior of a planned system. The realization of a physical demonstration system is therefore a very important tool to help in the discussion of the solution and also a catalyser for gathering precise definitions of requirements and to refine solutions. This unit can therefore support the development of demonstrating solutions jointly developed by researchers and enterprises. It is expected to involve in a later stage, research people from trade unions, and operators that can perform some demonstration tasks. The demonstration unit has an architecture in which it can be operated either as an integrated FMS/FAS system or as a set of isolated subsystems (machining, assembly, transportation and storage, etc.). This last aspect has particular consequences on the design of the control architecture.

\section{CONCLUSION}

The evolution of manufacturing systems towards Complex Manufacturing Environments raises new challenges in the technological and organisational innovation processes. In particular, methodological, theoretical and practical issues related with interdisciplinarity must be well understood and solutions devised aiming at effectively undertake techno-organisational projects. In this paper we outlined one possible approach to the study of such development methodologies, trying to balance the contributions of the Computer and Social Sciences disciplines. This approach will be materialised in a $\mathrm{R} \& \mathrm{D}$ project but, due to the underlying philosophy, it would be much more effective as an on-going live laboratory trying to cope with the continuous technological and social progress by assessing and developing new paradigms. 


\section{REFERENCES}

De Sitter, L. and Den Hertog, J. (1990). Simple organizations and complex jobs: the dutch socio-technical approach. In Annual Conference of the Academy of Management.

Latniak, E. (1995). The Symbiosis of Work and Technology, chapter 'Technikgestaltung' (Shaping of Technology) and Direct Participation; German Experiences in Managing Technological Change. Taylor \& Francis.

Riis, J., Johansen, J., and Mikkelsen, H. (1993). Games in production management. In Papas and Tatsiopoulos, editors, Advances in Production Management Systems (B-13). Elsevier Science Publishers.

Williams, T. E. a. (1993). Architectures for integrating manufacturing activities and enterprises. In Yoshikawa and Goossenaerts, editors, Information Infrastructures Systems for Manufacturing (B-14). Elsevier Science Publishers.

\section{BIOGRAPHY}

António Brandão Moniz is a Sociologist, Professor of Industrial Sociology and Sociology of Organisations at the Faculty of Sciences and Technology of New University of Lisbon (FCT-UNL), and researcher at UNINOVA-CRI. The main research interests are the human factors and the social implications of industrial automation, and the qualification aspects in manufacturing change.

António Lucas Soares is an Electrical Engineer teaching at the University of Porto and doing research work at INESC-ESP. He holds a MSc. in Industrial Automation and is currently undertaking its PhD work in the area of collaboration models for techno-organisational development. The main research interests are the interdisciplinary knowledge representation, techno-organisational development of integrated manufacturing systems and socio-technical systems. 